\title{
GRAVITATIONAL LENSES
}

\author{
Bernard F. Burke \\ Massachusetts Institute of Technology \\ Cambridge, MA 02139 \\ USA
}

\begin{abstract}
The gravitational lensing phenomenon is a new observational tool in cosmology. It appears to occur reasonably frequently; several thousand examples probably are observable with current radio and optical telescopes. A dozen or so reasonable candidates have been proposed, but it has become clear that one must apply strong tests to distinguish between real examples of lensing and chance associations. The dramatic double quasar $1146+111$ has emphasized the need to be rigorous, and data is presented that casts further doubt on its status as a lens. There are a number of well-established cases, however, and these illustrate the varied character of possible lenses. Present observational programs should enlarge the number of established examples. Time-delay measurements are also in progress that give a new class of cosmological test; the original lensed quasar pair $0957+561$ seems to have a differential time delay provisionally estimated at $600 \pm 200$ days for the two principal images.
\end{abstract}

\section{GRAVITATIONAL LENS PROPERTIES}

The large-scale structure of the universe is influenced primarily by the gravitational force, but few observables are accessible to accurate observation. Acceleration of matter by gravity over cosmological distance and time is only indirectly observable; Hubble's program to infer acceleration on the largest scale by observing the change in the Hubble expansion with redshift is yet to be demonstrated conclusively. Even the local value of the Hubble constant is in dispute, and although it is generally agreed that the value must be of the order of 50 to $100 \mathrm{~km} / \mathrm{s} / \mathrm{Mpc}$, the uncertainty is an impediment to testing cosmological theory. The classical means of observing the magnitudes, sizes, redshifts, and clustering of galaxies remains the principal observational tool, using the largest telescopes, long observing times, and accumulating ever-improving data with great persistence. Thus, each new addition to this limited arsenal of observational means must be welcome. The gravitational lensing phenomenon, although proposed half a century ago by Zwicky (1939), became an observational reality when Walsh, Carswell, and Weymann (1979) showed that the radio source $0957+561$ was identified with a twin quasar that could be interpreted most directly as the double image of a single quasar, split by 6 arc-sec through lensing by an intervening massive object. Stockton (1980) and Young et al (1980) showed that there was a foreground cluster of galaxies, with the brightest member of that cluster only 


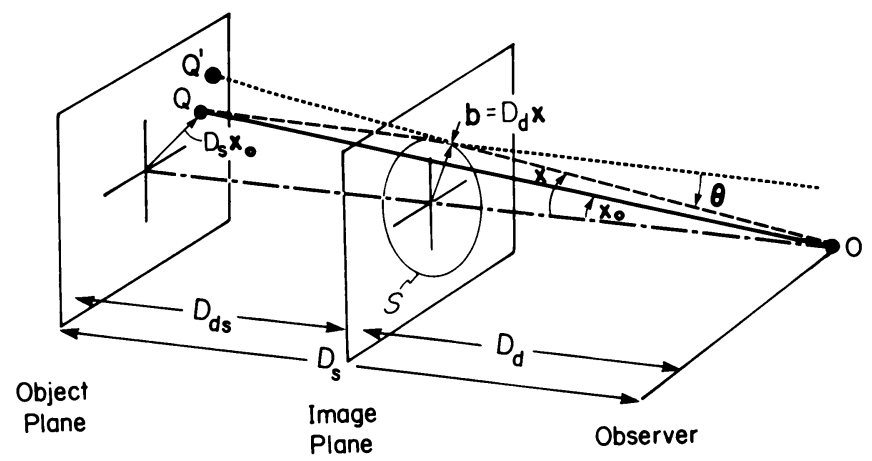

Figure 1: Gravitational Lens Geometry

1 arc-sec removed from one of the quasar images. In fact, that foreground object (a giant $\mathrm{cD}$ galaxy) would have had to produce multiple images in any event, and the proof was therefore conclusive. The analysis of VLA radio maps by Greenfield et al (1985) established conclusively that the lensing could not be produced by assigning a uniform mass-to-light ratio to the foreground galaxies, or even by a luminosity-dependent $M / L$, and that a substantial quantity of dark matter in the lens had to be invoked.

A number of recent reviews of gravitational lensing have appeared; the review by Canizares (1987), the theoretical reviews by Peacock (1983), Gott (1985) and Narayan (1986) and the observational reviews by Turner (1985) and Burke (1986) can be referred to. Most of the treatments so far have concentrated on the single-lens case, in which a distribution of matter at distance $D_{d}$, represented as a surface density $\sigma(\mathbf{x})$ (since the deflection angle is small), bends the light from a distant object, represented by a brightness distribution $B_{o}\left(\mathbf{x}_{0}\right)$ at distance $D_{s}$, where $\mathbf{x}_{0}$ are the angular coordinates in the object plane constructed at the source. The image plane is usually constructed through the lens, and the image is seen in projection with angular coordinates $\mathbf{x}$, with the original (undeflected) brightness distribution $B_{o}\left(\mathbf{x}_{0}\right)$ transformed to an apparent brightness distribution $B_{i}(\mathbf{x})$. The action of the lens can be described by the gravitational deflection $\Theta(\mathbf{x})$, the angle by which a ray is deflected when passing the lens center at an impact parameter $\mathbf{b}=D_{d} \mathbf{x}$. The geometry is shown in Figure 1 .

In the lensing calculations, the quantity $D_{*}=D_{d} D_{s} / D_{d s}$, the effective lensing distance, is a natural parameter. Distance, in the cosmological sense, must be defined carefully; Press and Gunn (1973) address the question, and Alcock and Anderson (1985) examine the complications that arise in a universe composed of discrete massive objects 
(see also the review by Hewitt, 1986). For a homogeneous Friedman universe, angular size distance is the appropriate measure; if the universe is so inhomogeneous that there may be no matter along the immediate line of sight, the subtleties explored most recently by Ehlers and Schneider (1986, henceforth ES) must be heeded. These considerations enter the discussions of Sections 5 and 6 , and illustrate the broadening of cosmological questions that arise when studying gravitational lenses.

The lensing transformation preserves specific intensity unless the lens has a large motion with respect to the local Hubble flow, so the transformation $B_{o}\left(\mathbf{x}_{o}\right) \rightarrow B_{i}(\mathbf{x})$ is equivalent to the coordinate transformation $x_{0} \rightarrow \mathbf{x}$, and the calculation can proceed either by ray-tracing for the given gravitational deflection law $\Theta(b)$, or Fermat's principal can be invoked by making a variational calculation of the time delay for all possible ray-paths from a given $x_{0}$ and looking for extrema. Ray-tracing was used in the early calculations, but Fermat's principle, introduced by Schneider (1984) and explored further by Blandford and Narayan (1986) gives direct insight into the image topology (see also the reviews by Narayan (1986) and by Canizares (1987)).

The two elementary examples, the compact (point) mass $M$ and the singular isothermal sphere, of velocity dispersion $\sigma$ are easily solved in analytic form. When the lens and the source are co-aligned, $x_{o}=0$, and the image is a ring of radius $\alpha_{o}$ :

$$
\alpha_{o}= \begin{cases}\left(2 R s / D_{*}\right)^{1 / 2} & \text { (point mass) } \\ 4 \pi(\sigma / c)^{2}\left(D_{d} / D_{*}\right) & \text { (singular isothermal sphere) }\end{cases}
$$

Note that the Schwarzschild radius for the mass $M\left(R s=2 G M / C^{2}\right)$ enters for the point mass case, while the angle of deflection for the singular isothermal sphere is constant and depends only on the velocity dispersion. The critical radius $\alpha_{0}$ is a useful parameter to estimate lensing effects for simple cases. For example, for an object quasar at $3 \mathrm{GPc}$, with the lens at half the distance $\left(D_{*}=2 D_{d}\right)$, consider the effects of the two simple lens models described above. If the lens is a compact massive object of $10^{11} M_{\odot}$ the pointmass case applies, while a singular isothermal sphere with $\sigma=250 \mathrm{~km} / \mathrm{s}$ is a reasonable approximation to a galaxy; for these cases the critical angle $\alpha_{o}$ is 0.2 and 0.5 arc-sec, respectively. Modifications of the isothermal sphere are frequently used to model galaxies. (Bourassa and Kantowski (1975) showed how the treatment of the sphere generalized to the ellipsoidal case.)

When there is a slight misalignment, the ring breaks into two images, one outside $\alpha_{0}$ and magnified, and the other inside $\alpha_{0}$ and demagnified. As the angle of misalignment increases, the outer image approaches unity magnification and $x=x_{0}$, while the inner image goes to the center and diminishes to zero. A real mass distribution is unlikely to have singularities, and for the spherical case there will be a third image (and possibly more). The number of images must be odd (Dyer and Roeder, 1980 and Burke, 1981) for any non-singular mass distribution in the lens. With increasing misalignment ( $x_{o}$ increasing) the images will merge. In the three-image case, there will always be a maximum locus at which the images merge leaving only the principle image, and in general there will be a set of such caustic surfaces separating zones of different multiplicity.

The most common measurement so far has been the observation of the redshift and flux distribution: optically, one sees the multiple images of the quasar and (sometimes) the lensing foreground object. The total magnification is sampled at the locations of the quasar images, but since the original flux and position is unknown, only the flux ratios and relative image locations can be observed. Analysis proceeds by modelling the lens, which is not a unique process in general. The radio measurements can observe extended structures, in many cases, and since the magnification is a symmetric tensor, one can in principle observe both the longitudinal and transverse magnification, plus the the orientation of the 
principal axis (relative from one image to another). The more complex the object, the more rigorous the constraints that can be placed on the allowable models. In this respect, radio-loud quasars (and radio galaxies, although these are more difficult to disentangle) are to be preferred. Optical observations, of course, are always needed for the redshift information.

Quasars vary their flux with time, and since there will be different time delays for each quasar image, the cross-correlation of quasar image fluxes should yield a new class of cosmological observable. Cooke and Kantowski (1975) gave the formalism, in which the time delay is the result of two effects, the "geometric" time delay obtained from ray tracing and the "gravitational" delay from the passage of the rays through different parts of the gravitational potential well of the lens (both being one effect in the proper GR treatment). The definition of "distance" is again relevant. If the universe is sufficiently homogeneous, the time delay depends on the Hubble constant and the lens model, but a non-zero cosmological constant or a "lumpy" universe was shown to have a measureable effect on the time delay by Alcock and Anderson (1985). The interesting prospect arises, therefore, that apparent values of $H_{0}$ might be determined through time delay measurements that differ from that obtained by the photometric methods in common use. Because the time delay method is sensitive to these perturbations one can invert the argument and say that measurements of $\Delta t$ are an excellent way to place limits on $\Lambda$ and on the inhomogeneity of the matter distribution in the universe. The present status of time delay measurements is described in Section 6. The time delay observations can be complicated by "microlensing" from the stars in foreground galaxies. This can be a real concern for optical measurements of highly compact sources such as optically violent variables, but is not likely to be important for radio measurements. The review of Canizares and the recent work of Paczynsky (1986) can be referred to.

The statistical properties of gravitational lenses may have direct relevance to the "dark matter" problem. The first need is to establish a reliable list of gravitationally lensed objects; this is addressed in Sections 3 and 4. Given a reasonable sample of objects, which is the aim of the MIT-Princeton gravitational lens search described by Hewitt et al (1987a), the probability of a given class of lensing occurrence allows one to approach the question of how mass concentrations are distributed in the universe. Even the limited data available so far allows limits to be set, and these are described in Section 5.

The review of Canizares (1987) emphasized two long-standing problems, the "missing image" and the "elusive lens". These can be set aside for the moment, but not ignored completely. It is an inescapable fact that most of the instances of lensing exhibit an even number of images, when the number should be odd. The effect can be understood if each lens has a sufficiently large and compact mass at its core (Narasimha, Subrananian, and Chitre, 1986), and ultimately the search for the final image may have relevance to the general question of the nature of galactic nuclei. The class of apparent gravitationally lensed quasars that show no foreground lensing candidate, the "elusive lens" problem, underlies much of the discussion in Section 3 and 4; as Gott (1987) emphasized in the IAU Symposium on "Dark Matter in the Universe" (Kormendy and Knapp, 1987), these may be the only direct way of measuring the distribution of that important but hidden component of the Universe. There is a strong burden of proof on the observer, however, to show that such cases are true instances of lensing.

\section{LENS IDENTIFICATION: THE CASE OF 1146+111}

Initially, gravitational lensing was identified by observing that close sets of quasar images exhibited the same redshift. The first instance of lensing, $0957+571$, met this 
criteria well. After a brief uncertainty that stemmed from the unusual form of the radio image, the work of Stockton (1980) and Young et al (1980) clarified the matter. The " $B$ " quasar image, 6 arc-sec $S$ of the " $A$ " image, was accompanied by a giant elliptical galaxy 1 arc-sec $\mathrm{N}$, and since the quasars exhibited redshifts of about 0.4 , it was clear that lensing was inevitable. If a quasar is aligned that closely with a galaxy as massive as G1, the appearance of other images is inevitable if the same physical laws govern different parts of the universe, as we believe they do. The galaxy G1 was the brightest member of a rich cluster, and it was not surprising that the mass distribution of G1 alone could not explain the observations. What was more remarkable was the quantity of matter not associated with G1 that was needed to explain the radio observations. Young et al (1981) examined a double-ellipsoid model that gave a reasonable approximation to the data; one ellipsoidal mass distribution represented the galaxy G1, while the second represented the mass associated with the cluster of galaxies as a whole. The models required a very large mass for the second component. Further studies were made by Higgs (1984), Greenfield et al (1985), and Roberts et al (1985), and the conclusions were clear. The radio data could be only satisfied by a mass distribution that was inconsistent with assuming constant massto-luminosity for the foreground galaxies. The large amount of matter that was needed had to be in the form of "dark matter". Since clusters of galaxies in general exhibit large velocities that imply, through the virial theorem, that a dark matter component is the dominant mass fraction, the result for $0957+561$ was not unexpected. The most significant development would be that a gravitational lens might be found that had a sufficiently complex radio pattern to permit sharper limits to be placed on the distribution of the dark matter component. The subsequent discovery of two examples of lensing, 1635+267 and $2345+007$, that exhibited double quasar images with no apparent foreground lensing galaxy raised the expectation that complexes of dark matter with no associated luminous matter were a reasonably frequent occurrence.

The subject reached a critical stage when Turner et al (1986) reobserved the widespacing double quasar $1146+111 \mathrm{BC}$, discovered by Hazard et al (1979) and recognized as a pair with nearly identical redshifts of 1.01 by Arp and Hazard (1980). The quasar pair are members of a group of quasars having various redshifts; the letter designations follow the second publication. The strikingly similar spectra and redshifts obtained by Turner et al suggested that it was a lensed pair, but the separation of 157 arc-sec, two orders of magnitude larger than commonly observed in previous examples of lensing, raised the possibility that a new phenomenon was at hand, perhaps a cosmic string (Vilenkin, 1984, Paczynski, 1986) or a supermassive black hole (Gott, 1986). Shaver and Christiani (1986) showed, however, that the infrared characteristics of the pair were not identical, concluding that the pair might be separate quasars, probably in the same cluster. Subsequently Phinney and Blandford (1986) and Bahcall, Bahcall, and Schneider (1987) argued that a modest degree of quasar clustering might give a few such examples in the visible sky. The lensing explanation, therefore, while not eliminated, clearly needed stronger proof.

VLA observations were undertaken, and Langston et al (1986) concluded that the radio field provided no substantiating evidence that either a massive black hole or a cosmic string is responsible for the image pair. The $6 \mathrm{~cm}$ VLA map shown in Figure 2 shows the basic data. The quasar pair, $\mathrm{B}$ and $\mathrm{C}$, are shown by the two crosses, and since the upper limit to their radio fluxes is $\mathbf{1 5 0}$ microJy, both are clearly radio quiet. The field, as noted by Arp and his colleagues, contains many quasars, two of which are radio loud: $A(z=$ $0.86)$ and $\mathrm{D}(\mathrm{z}=2.12)$. A "conventional" cosmic string, if it is not moving at relativistic velocity, should have given a companion image of $D$ that is not seen. A supermassive black hole would have to be situated roughly half way between $\mathrm{B}$ and $\mathrm{C}$, and the flux of $\mathrm{D}$ is large enough to require a companion image to be seen to the right of the $\mathrm{BC}$ line. No 


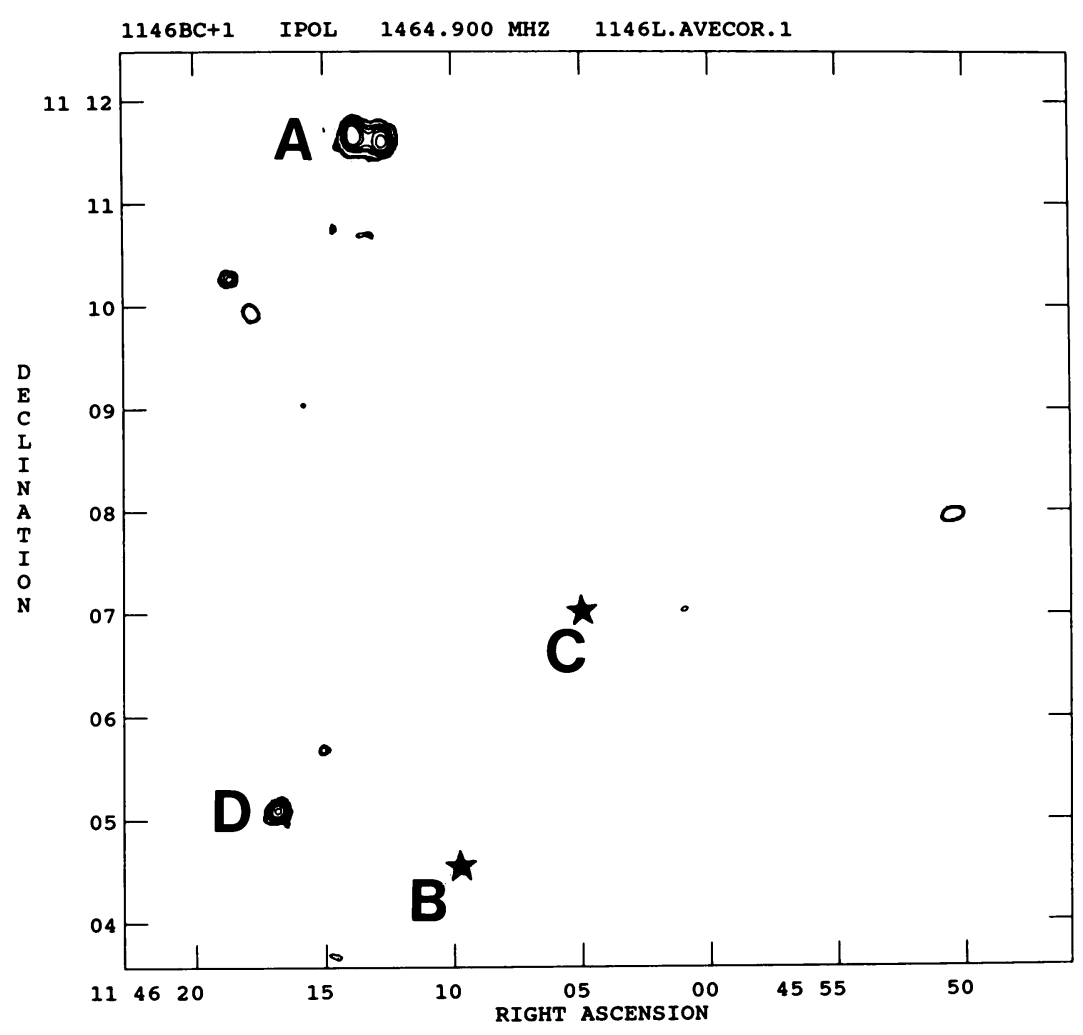

Figure 2: $\lambda 6 \mathrm{~cm}$ field of $1146+111$, showing the locations of the quasars $B$ and $C$, with $A$ and $D$ also identified. The contour levels are $0.15,0.3,1.0,3.0,10,30,100 \mathrm{mJy}$. 
such radio image is visible. One can, of course, invoke time variability, but the relative time delay of several hundred years is unlikely to be large enough for quasar $D$ to have suffered such a major fluctuation. There are lens models that would be consistent with the lack of a counterpart image for $D$, such as a singular isothermal sphere whose caustic includes $\mathrm{B}$ but excludes $\mathrm{D}$, but the general conclusion is inescapable: Figure 2 contains no evidence that $B$ and $C$ are lensed images of a single quasar.

The likelihood that pairs of quasars may mimic the lensing phenomenon raises the question of which lens candidates are truly examples of lensing, and which are only associated pairs either by chance or by being in the same cluster. Here, the advantages of concentrating on radio-loud quasars becomes apparent. The optical observables consist of source position, source fluxes as a function of time, radial velocity, and spectral characteristics. The radio data include, if one is fortunate, a more complex large-scale image distribution and microstructure on the milliarcsecond scale associated with the core of each image. The optical images are indispensable for radial velocity and spectral information, of course. Spectral similarity is not enough, as Shaver and Christiani have shown; quasars have generic similarities in many cases.

\section{CRITERIA FOR LENSING}

Table 1 shows the current roster of lensing candidates. These have been examined in the light of the following criteria:

(1) Observable foreground lens that should produce multiple images

(2) Microstructure on the milliarc-second scale consistent with lensing

(3) Correlated time-delay observations

(4) Identical redshifts

(5) Large-scale structure compatible with lensing

(6) Similarity of spectra

In each case, there will be degrees of certainty but there are examples that pass several of the tests easily. The existence of a foreground lens (Criterion 1) can stand alone, in some, cases, as can the existence of similar microstructure (Criterion 2). The latter, however, needs qualification. There are quasars that exhibit rapid motion in the core, sometimes apparently in excess of the speed of light. Since there will be a relative time delay between the images, they will not look the same, although their development in time should be similar. Even this might need qualification if the core appearance varies rapidly with viewing angle, but the angular differences are small; the danger of such an occurrence is probably slight.

Criterion (3), the observation of similar time variations of the components but with a time lag from one to another, is clear verification that one is dealing with a gravitational lens. There are two potential limitations: firstly, the data are severely aliased in practice because of observing limitations; secondly, the effects of microlensing may comlicate the time variations, especially at optical wavelengths. Radio flux variations are generally immune from this complication.

The fourth criterion, identicality of redshifts, is a less reliable guide, although in those cases where the redshift accuracy can be narrowed to a few tens of kilometers per second, the proof is reasonably strong. A pair of quasars in the same cluster might have redshifts that differ by several hundred $\mathrm{km} / \mathrm{sec}$, but for an ensemble of many lenses, 
differences of $50 \mathrm{~km} / \mathrm{s}$ or so would not be a probable occurrence. Unfortunately, the lensed quasars frequently are faint, and an uncertainty of a few hundred kilometers per second is often the best one can do. One should look for independent proof in such cases.

The appearance of the large-scale structure, particularly the radio noise distribution is a fifth criterion that in some sources could be absolute proof. The examples studied so far, however, have structure that is sufficiently complex to mask obvious lens-like properties. The double quasar $0957+561$ is a relevant example: there is a well-developed jet from $A$ that unfortunately lies mostly outside the three-image caustic. If this had not been the case, the lens nature would have been obvious.

The final criterion, spectral similarity, is the most elusive at present. Shaver, Wampler, and Christiani (1987) have shown that spectra in the $L y \alpha-$ C IV region and in the MgII 2800 region (unredshifted) can be remarkably similar from one quasar to another. There is a dearth of quantitative spectroscopic information on quasars, and the judgment as to whether a quasar pair have similar but uncommon spectra is generally subjective. One notes that differences in spectra may occur for lensed images, but if such differences exist a plausible explanation must be provided. These might include spectrum contamination from a foreground object, as in $0957+561$, or it could be caused by different microlensing of the images, with the compact, narrow-line features being more affected than the broad-line components by the different microlensing environments in a foreground galaxy.

These six criteria have been listed in approximate order of their persuasiveness. The first three are the strongest, and any one of these could stand alone as a test of lensing if the data were sufficiently unambiguous. The lack of one of these proofs would not be fatal necessarily; there may well be isolated concentrations of dark matter, for example, that would result in lensing with no visible foreground object. The lack of timedelay effects, similarly, could be explained by microlensing, although the radio fluctuations would probably be immune from such effects.

The final three criteria, in contrast, are apparently less reliable, although not without value. The study of quasar pairs will certainly lead to a better understanding of quasar clustering tendencies, and sufficiently accurate redshifts can still be convincing evidence for lensing. Only the last criterion, spectral similarity, is currently in doubt. The conclusion must be that all criteria will seldom be satisfied; failure of one or another test may not be fatal, if the effect has a reasonable explanation.

Table 1: Gravitational Lens Candidates, June 1987

\begin{tabular}{|c|c|c|c|c|c|}
\hline lens & $Z_{0}$ & $\left(Z_{L}\right)$ & $N_{i m}$ & $\alpha(\operatorname{arcsec})$ & Verification ${ }^{\dagger}$ \\
\hline 1. $0957+561$ & 1.4 & $(0.39)$ & 2 & 6 & $1,2,3,4,5,6$ \\
\hline 2. $1115+080$ & 1.7 & $(0.8 ?)$ & 4 & 2 & $1,3,4,5,6$ \\
\hline 3. $2016+112$ & 3.3 & $(\sim 0.8)$ & 3 & 4 & $1,4,5,6$ \\
\hline 4. $2237+031$ & 1.7 & $(0.04)$ & 2 & 2 & 1,4 \\
\hline 5. UM673 & 2.7 & $(0.49)$ & 2 & 2 & $1,4,6$ \\
\hline 6. $3 \mathrm{C} 324$ & 1.2 & $(0.84)$ & Ext. & 2 & 1 \\
\hline 7. $0023+171$ & 0.95 & - & $2+$ & 5 & 4,6 \\
\hline 8. $1635+267$ & 2.0 & - & 2 & 4 & 4,6 \\
\hline 9. $2345+007$ & 2.2 & $\cdot$ & 2 & 7 & 4,6 \\
\hline 10. $1042+178$ & 0.9 & - & 4 & 2 & \\
\hline 11. $2018+102$ & $0.62 ?$ & $(?)$ & 5 & 6 & \\
\hline 12. $2113+100$ & $1.864 ?$ & 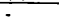 & 3 & 6 & \\
\hline 13. $1146+111$ & 1.0 & - & 2 & 157 & $4,6 ?$ \\
\hline 14. $0107-025$ & 0.96 & . & 2 & - & \\
\hline
\end{tabular}

References: (1) Young et al (1981); (2) Vanderreist et al (1985); Henry and Heaney (1986); (3) Schneider et al (1985, 1987); (4) Huchra et al (1985); Tyson and Gorenstein (1985); (5) Surdey et al (1987); (6) Djorgowaki and Spinrad (1984); (7) Weedman et al (1982); (8) Hewitt et al (1987); (9) Hammer et al $(1987)$; $(10,11,12)$ Hewitt $(1986)$; (13) Turner et ad (1986), Shaver and Chriatiani (1986), Langston et al (1986); (14) Surdey et al (1986) 


\section{LENS CANDIDATES: CURRENT STATUS}

The lens candidates in Table 1 have been divided into four groups: those that are certainly lenses, those that are probably lenses, those that look promising but with no definitive evidence yet, and those that are probably not lenses, but for which the clinching evidence is not yet in.

The wide-spacing double, for example, appears in the fourth category. It may yet turn out to be consequence of lensing, but the present evidence is weak. Other evaluations follow.

$0957+561$ This, the original double quasar, was well-established as a lens by the optical work summarized by Young et al (1981) and by the radio work of Greenfield et al (1985) and Roberts et al (1985). VLBI studies by Gorenstein et al (1987) have, in the meantime provided a dramatic confirmation. The central structures of the $A$ and $B$ images both show a compact core and jet with a total extent of less than ten milliarcseconds, accompanied by a more extended jet of order 50 mas in length. The extended jets are asymmetrical, and show odd relative parity, as they should according to the standard double-ellipsoid model. More generally, the Fermat-principle-derived predictions of Blandford and Narayan predict the same result. Time delay observations are reported in Section 6. Extensive modelling studies have been carried out on this source. In addition to the work of Young, et al, Higgs, and Greenfield et al, Greenstein, Falco, and Shapiro (in preparation) have looked at problems of parameter estimate degeneracies. Their recent study of $0957+561$ (Falco et al, 1987) has emphaseized the importance of determining the velocity dispersion of $\mathrm{G} 1$ if $H_{o}$ is to be estimated from the relative time delay $\Delta t(A, B)$.

$1115+080$ Originally known as the "triple quasar", the model of Young (1981) predicted that it should be quintuple, and that the bright $A$ image should be double. Hege (1981) demonstrated the latter, and Young's model predicted that the fifth image should be faint, so there is no inconsistency. Young predicted that there should be an edge-on massive galaxy in the association. While Henry and Heaney (1986) have detected a foreground galaxy, it is in the midst of the C-image complex contrary to Young's predictions. The system is radio quiet at the $50 \mathrm{mJy}$ level (Burke et al, unpublished). The multiple character meets condition (5), the components as a whole meet condition (4), and there is a foreground galaxy that probably satisfies (1). More particularly, Young's prediction was a strong one, made a priori, and the system is certainly an example of lensing.

$2016+112$ This trio of radio sources was established as a lens at an early stage by strong positive evidence conforming to criteria (4) and (6). The optical spectra of the $A$ and $B$ quasar images are unusual, with extraordinarily sharp lines that permit accurate determination of the redshift equality. The recent study of Schneider et al (1987) showed that near the third radio object $C$ there is a third quasar image; this complex, therefore seems to have the distinction of being the only instance of an odd number of images. VLBI studies (Gorenstein, Heflin et al, in preparation) show that the $A$ and $B$ images have similar structures at the milliarcsecond level, but current $A$ and $B$ models are not consistent with zero time delay gravitational lensing. There are two foreground galaxies that undoubtably contribute to the lensing, but no accompanying cluster. The data of Schneider et al $(1985,1987)$ show that galaxy $D$ is at $\mathrm{z} \sim 1$ (the most distant known radio-quiet giant elliptical). Galaxy $C$ is mixed with the image of the third quasar image, and its photometric redshift, $\mathrm{z} \sim 0.8$ is consistent with that of $D$. Lensing models have been examined in greater detail by Narasimha et al (1987), who suggest that it is actually a five-image configuration with two merging images rather than just three images, perhaps analogous to the case of $1115+080$.

$2297+031$ The lens quality derives from condition (1). Huchra et al (1985) saw that 
the nucleus of a $15 \mathrm{~m}$ galaxy exhibited two redshift systems, one at $z=0.04$, consistent with the bright galaxy, and an emission-line system at the typical quasar redshift $\mathrm{z}=$ 1.70. Chance alignment seemed improbable, unless there was gravitational magnification. The hypothesis was supported by Tyson (1986) who saw a trio of images, presumably the galaxy nucleus and the two brightest quasar images, and confirming optical observations of high quality have been made in the course of the Princeton-MIT lens study (Turner, private communication).

$0023+171$ This is an example of the second class of lens in Table 1, promising but not certain. The study by Hewitt et al (1987) summarizes the present state of the evidence: two quasar-like but faint $(\gamma=23.4,22.8)$ optical images. The brightest optical object corresponds in the radio to an unresolved source $C$, while the fainter optical object is in the midst of a more complex double radio source, $A B$. There is no significant evidence for a foreground lensing galaxy, although the extended radio emission might be related to a relatively faint radio galaxy. The radial velocities of the optical components are equal to within $150 \mathrm{~km} / \mathrm{s}$. The conclusion of Hewitt et al, that the combination of low redshift $(\mathrm{z}=0.95)$ faintness of any possible foreground object, and the large separation ( 5 arcsec) implies a large mass-to-light ratio (of order $10^{3}$ ) depends upon the correctness of the lensing interpretation.

UM679 The two images of this pair have practically identical redshifts and the spectra have detailed similarity. The continuum difference between the images shows a small continuum step that is consistent with a $\lambda 4000 \AA$ break redshifted by $\mathrm{z}=0.49$.

3C32 4 This system is probably the first example of a radio galaxy undergoing significant distortion by a foreground galaxy. It remains to be proven that the radio source has distortion consistent with lensing, but if the foreground galaxy can be proven to be a large system (the emission lines in its spectrum suggest this) then by criterion (1) it meets the necessary tests.

$0028+171$ This is an example of the the second class of lens in Table 1, promising but not certain. The study by Hewitt et al (1987) summarizes the present state of the evidence: two quasar-like but faint $(\gamma=23.4,22.8)$ optical images. The brightest optical object corresponds in the radio to an unresolved source $C$, while the fainter optical object is in the midst of a more complex radio source, $A B$. There is no significant evidence for a foreground lensing galaxy, although the extended radio emission might be related to a relatively faint radio galaxy. The radial velocities of the optical components are equal to within $150 \mathrm{~km} / \mathrm{s}$. The conclusion of Hewitt et al, that the combination of low redshift $(z=0.85)$ faintness of any possible foreground object, and the large separation ( 5 arcsec) implies a large mass-to-light ratio (of order $10^{3}$ ) depends upon the correctness of the lensing interpretation.

$1635+267,2945+007$ Both of these double quasars lack a visible lensing galaxy or cluster in the foreground. Both are faint objects, and obtaining either time-delays or sufficiently accurate redshifts and spectra to convince one that they are truly lensed pairs is a challenging but feasible task. Neither are radio-loud above the $50 \mathrm{mJy}$ level, so the proof must rest on optical data alone.

$1042+178,2018+102,2113+100$ These show compact, multiple radio and optical structure, but the detailed spectroscopic data has not yet been taken.

$1146+111,0107-025$ These two wide-spacing double quasars seem to have only a remote chance of being examples of lensing. The first was described at length in Section 3; the second, originally proposed by Surdej et al (1983), has been studied further by the same group ( Surdej et al, 1986). The two quasar spectra are indeed remarkably similar, but they report a real difference in redshift of about $1200 \mathrm{~km} / \mathrm{s}$. A rapidly moving lens or cosmic string could produce such an effect, but confirmatory evidence from other field 
objects has not been produced. One should reserve a shadow of a possibility that these are lensed pairs, but the evidence against that interpretation is accumulating.

\section{LENS STATISTICS}

The probability that a given quasar or radio galaxy will undergo significant lensing depends not only on the characteristics of galaxies, clusters, and aggregations of dark matter, but on the large-scale structure of space and time. Propagation in a smooth, Friedman-class universe can be treated by standard methods, but when the matter is distributed in a clumpy fashion, more care must be exercised. The probability that detectable lensing would occur was calculated starting from the work of Dyer and Roeder (1972), by Press and Gunn (1973) who were interested in whether massive black holes might distort the appearance of distant galaxies. A more extensive analysis, following the actual demonstration by Walsh et al that lensing occurs, was carried out by Turner, Ostriker, and Gott (1984, henceforth TOG). In their analysis they assumed that individual galaxies could be modelled as singular isothermal spheres (they also considered point masses) and the probability of lensing of distant quasars was considered for several cosmological models. Their principal conclusion was that if multiple imaging was much less frequent than once per five hundred quasars, one or more generally held beliefs concerning cosmological models or galactic structure would need major revision. They also showed that the most probable redshift for a lensed quasar would be approximately $\mathrm{z}=1$, and that the most probable lens redshift would be $z=0.5$. They also demonstrated that their predicted distributions of image separation did not resemble the observed distribution, since all known examples had separations of several arc-seconds, in contrast to their most probable separation of half an arc-second or so. They recognized that observational selection might be responsible for the dearth of close-spacing pairs, and that dark matter concentrations in clusters of galaxies might modify the predictions substantially.

The MIT-Princeton lens survey, outlined by Hewitt et al (1987), is several years from completion but enough VLA maps have been examined to allow some statistical statements to be made. By applying the formalism of functions of a random variable, Hewitt (1986) generalized the TOG study, by setting up the mechanism for constructing the probability distribution $d P(Q)$ for any measurable quantity $Q$ in a given cosmological model. The angle of separation between pairs, $\alpha_{o}$, and the flux ratio $R$ are the most readily measured examples of a particular $Q$, and were used by Hewitt as specific examples. The instrumental limits imposed by the VLA were included explicitly in calculating the distribution of flux ratios and image separations. The probability distributions are therefore directly comparable to the VLA observations. The VLA observations were carried out in both the $A$ and $B$ configurations at $\lambda 6 \mathrm{~cm}$, with angular resolutions of 0.3 and 1.0 arc-sec. The range of observability of double sources that meet the particular observing criteria are plotted in Figure 3a as a function of flux ratio versus image separation (Rvs. $\alpha$ ). The particular observing parameters translated into limits of 0.4 to 8.3 arc-sec separation for $R>2$, and 0.4 to 64 arc-sec for $R<2$. The limits of observation for the A-array in an $R-\alpha$ representation are shown in Figure 3a, which shows that image separation between 0.4 and 8.3 arc-sec are detectable with certainty if $R>2$, and between 0.4 and 64 arc-sec if $\mathrm{R}<2$. For a particular class of lens these limits map into a representation in the $\alpha-\beta$ plane, where $\alpha$ is the (observed) image separation and $\beta$ is the (undeflected and unobserved) position $x_{o}$ of the object quasar. Figure 3b shows the limits of Figure 3a, mapped into the $\alpha-\beta$ plane for a point mass lens, while Figure 3c shows the same mapping for an isothermal sphere lens. The probability calculation depends on determining the upper 
and lower limits of the allowed values of $\beta$ for a given $\alpha$, and preceeding to ensembles of lenses in order to generate $d P(\alpha)$ and $d P(R)$ for given cosmologies and lens models.

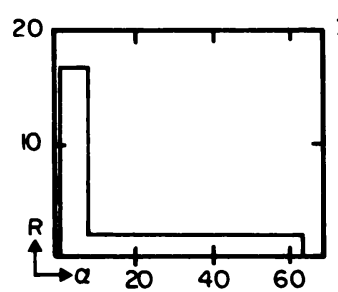

(a)

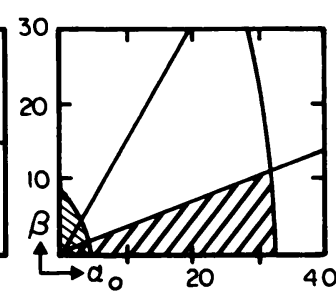

(b)

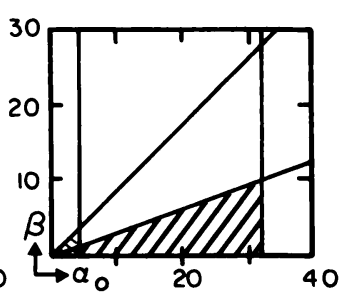

(c)

Figure 3: (a) VLA observing limits on the image separation-flux ratio plane $(\Delta a, R)$. In (b) and (c) these are mapped onto the critical angle-original position plane $\left(\alpha_{0}, \beta\right)$, with (b) being the point-mass case and (c) being for singular isothermal spheres. All angles are in arc-seconds.

The most conservative assumption in applying this formalism to the data is to ascribe all examples of double structure to lensing. This is overly conservative, because most such structures are double-lobed radio galaxies, but it certainly leads to a rigorous upper limit to the space density of lenses. The conclusion of the Hewish study was that all lensing events caused by compact masses in the range $10^{11}$ to $10^{13} M_{\odot}$, or by isothermal spheres with velocity dispersions in the range 210 to $380 \mathrm{~km} / \mathrm{s}$, would be detected by the VLA in its $A$ configuration. Furthermore, since the shape of the distribution of flux ratios is preserved in the VLA observations, both the source separations and the flux ratios are appropriate observables. A number of calculations were made of the limits that could be placed on the fraction of lens-derived mass $\Omega_{L}$ represented by various populations of compact masses and isothermal spheres, for total mass parameters $\Omega=0$ and $\Omega=1 \quad(\lambda=$ $0)$ Friedman cosmologies. For point masses in the range $10^{11}$ to $10^{13} M_{\odot}, \Omega_{L}$ cannot exceed 0.4 , and for isothermal spheres in the range 210 to $380 \mathrm{~km} / \mathrm{s}$ the number density cannot exceed $3 h^{3}(\sigma / 300)^{-4} \mathrm{Mpc}^{-3}$. These are primarily illustrative of the promise of the method, and far more explicite upper limits on $\Omega_{L}$ will be placed when the survey work 
is complete.

The recent study by ES has examined the fundamental lensing process more closely, and in particular examines the limitations of the Dyer-Roeder propagation formalism. Hewitt (private communication) has compared the ES and TOG formalisms, and concludes that, to within the model assumptions, the ES formalism is superior since TOG underestimates lensing effects at high redshifts ( $\mathrm{z}$ of 2 or 3 ). Most of the lenses act on quasars closer to unity redshift, and the estimates based on TOG (such as the study by Hewitt) can still be used to set lower limits on lensing probability.

\section{TIME DELAY}

The relative flux variations of only two lens systems have been reported so far. The observations are not straightforward, because the optical observations are interrupted for half the year by the sun, while radio observations by the VLA are possible in the highresolution $A$ and $B$ arrays for only half the year as well. The radio interrruptions are not in synchronism with the solar year, since the cycle for array changes runs 16 months or so. The present status for each source follows.

$1115+080$ The observations of Vanderriest et al (1985) showed that the time delays between fluctuations in the pairs $(A, B)$ and $(A, C)$ are probably less than 2.5 months, while the time delay $\left(A_{1}, A_{2}\right)$ is probably greater than 4 days. These limits are not compatible with Young's original model, but are compatible with the later model by Narasinha et al (1982).

$0957+561$ The first observation of flux variations was reported by Florentin-Nielsen (1984), who reported a time delay of about 500 days (16 months) for the $(A, B)$ pair with $A$ varying first. Because of the limited time span of that data, this value needed confirmation. Schild (1985) reported a time delay of 400 days (1.1 years) based on a sharp fluctuation that occurred in the image fluxes, but this was a single-point correlation, and since the optical quasar flux varies greatly, it seemed advisable to conform this data also. The optical data are capable, in principle, of giving very accurate values of $\Delta t$ because the variations are rapid, but since the blocking time is one year, any value of $\Delta t(A, B)$ of the order of a year that is based on one or two years of data must be taken as provisional.

A series of ultraviolet observations was undertaken, using the IUE satellite, and a preliminary report on the value of $\Delta T(A, B)$ has been reported by Gondhalekar et al (1987). Eleven observations were made in the period 1980 to 1983, and it was clear that the source fluxes varied significantly from one observation to another. A dip in the flux of $A$ was followed some time later by a similar dip in the flux of $B$, and these give a formal time delay of 660 days $(1.8 \pm 0.2 \mathrm{y})$. As in the case of the ground-based optical observations, one would wish for a longer series of data, with the occurrence of several such events.

The radio data should give a more definitive value for the time delay, since microlensing is not a concern, but the flux variations at radio wavelengths are smaller than the optical variations, and occur more slowly. Hewitt, Roberts, and Burke have been using the VLA to monitor the flux variations $0957+561$ over the last seven years, and it seems advisable to give a progress report on this work, both to outline the problems and to stimulate confirmatory work by others.

The $\lambda 6 \mathrm{~cm}$ fluxes of both $A$ and $B$ diminished slowly, at about $1 \mathrm{mJy} / \mathrm{yr}$, from 1980 to 1985 . The $A$ and $B$ fluxes had been 35 and $25 \mathrm{mJy}$ at the beginning of that period, and had diminished to 29 and $19 \mathrm{mJy}$ by 1985 . The flux of $A$ then jumped to 31 mJy, while the flux of $B$ increased to $21 \mathrm{mJy}$. The data were cross-correlated, and until 
more data is obtained only a range of possible time delays can be stipulated, although the variations are highly reliable. A cross-correlation of spline fits yields a time delay of 600 days, while a Fourier analysis gives 500 days. Direct superposition of the two time series shows that a time delay of $\mathbf{4 0 0}$ days is barely acceptable, as is also the case for $\mathbf{7 5 0}$ days. The present conclusion is that the time delay is $600 \pm 200$ days. For a given lens model, this time delay can be used to derive a value of $H_{o}$, subject to the provisos of Alcock and Anderson. The model of Falco, Gorenstein, and Shapiro gives, for $\Delta T=600$ days, a value of $50 \mathrm{~km} / \mathrm{sec} / \mathrm{Mpc}$ for the Hubble constant.

\section{ACKNOWLEDGEMENTS}

The radio work reported here was carried out at the VLA, a facility of the National Radio Astronomy Observatory, operated by Associated Universitites, Inc. under contract with the National Science Foundation. Thanks are due to many colleagues who provided advice and counsel; I am particularly indebted to J.N. Hewitt, E.L. Turner, M. Gorenstein, and M.B. Heflin. This work was supported in part by a grant from the National Science Foundation.

\section{References}

[1] Alcock, C. \& Anderson, N. Astrophys. J. 291, L29 (1985).

[2] Arp, H.C. \& Hazard, C. Astrophys. J. 240, 726 (1980).

[3] Blandford, R., \& Narayan, R., Astrophys. J. 310, 568 (1986).

[4] Bourassa, R.R. \& Kantowski, R. Astrophys. J. 195, 13 (1975).

[5] Burke, W.L. Astrophys. J. 294 L1 (1981).

[6] Burke, B.F. Proc. IAU Symp. 119: Quasars (Swarup, G. \& Kapahi, V.K., eds.) Reidel (Dordrecht) 517 (1986).

[7] Canizares, C. Proceedings IAU Symp. 124: Observational Cosmology (Hewitt, A., Burbridge, G., \& Fang, L.Z., eds.) Reidel (Dordrecht) 729 (1987).

[8] Cooke, J.H. \& Kantowski, R. Astrophys. J. 195 L11 (1975).

[9] Dyer, \& Roeder, Astrophys. J. 174 L115 (1972).

[10] Dyer, \& Roeder, Astrophys. J. 180 L31 (1973).

[11] Dyer, \& Roeder, Astrophys. J. 238 L67 (1980).

[12] Djorgowski, S. \& Spinrad, H. Astrophys. J. 282, L1 (1984).

[13] Ehlers, J. \& Schneider, P. Astron. \& Astrophys. 168, 57 (1986).

[14] Falco, E.E., Gorenstein, M.V., \& Shapiro, I.I. Proc. IA U Symp. 129: The Impact of VLBI on Astrophysics and Geophysics (Reid, M.J., \& Moran, J.M., eds.) Reidel (Dordrecht, in press) (1987). 
[15] Florentin-Nielsen, R. Astron. \& Astroph. 138, L19 (1984).

[16] Foy, Bonneau, \& Blazit Astron. \& Astrophys. 149 L13 (1985).

[17] Gondalekhas, P.M., Wilson, R., Dupree, A.K., \& Burke, B.F. London Conference Proceedings 'New Insights in Astrophysics: Eight Years of Astronomy with IUE' European Space Agency (ESA) SP-263 (1986).

[18] Gorenstein, M.V. et al Proc. of IAU Symp. 129: The Impact of VLBI on Astrophysics and Geophysics (Reid, M.J., \& Moran, J.M., eds.) (in press) Reidel (Dordrecht) (1987).

[19] Greenfield, P.E., Roberts, D.H., \& Burke, B.F. Astrophys. J. 293, 370 (1985).

[20] Gott, J.R. Astrophys. J. 288, 422-427 (1985).

[21] Gott, J.R. Proc. IAU Symp. 117: Dark Matter in the Universe (Kormendy, J., \& Knapp, G.R., eds.) Reidel (Dordrecht) 219 (1987).

[22] Hammer, F., Le fevre, O., \& Nottale, L. et al IAU Symp. 124: Observational Cosmology (Hewitt, A., Burbidge, G., \& Fang, L.Z., eds.) Reidel (Dordrecht) (1987).

[23] Hege, E.K. et al Astrophys. J. 248, L1 (1981).

[24] Henry, J.P., \& Heaney, J.N. Nature 321, 139 (1986).

[25] Hewitt, J.N. Ph.D. Thesis, MIT (1986).

[26] Hewitt, J.N. et al Astrophys. J. 321, 706 (1987).

[27] Higgs, W.J. M.Sc. Thesis, University of Manchester, England (1984).

[28] Huchra, J. et al Astron. J. 90, 691 (1985).

[29] Langston, G. et al Bull. AAS 18, 930 (1986).

[30] Lawrence, C.R. et al Astrophys. J. 278, L95 (1984b).

[31] Narasimha, D., Subrananian, K., \& Chitre, S.M. MNRAS 200, 941 (1982).

[32] Narasimha, D., Subrananian, K., \& Chitre, S.M. Nature 321, 45 (1986).

[33] Narasimha, D., Subrananian, K., \& Chitre, S.M. Astrophys. J. 315, 434 (1987).

[34] Narayan, R. Proc. IAU Symp 119: Quasars (Swarup, G. \& Kapahi, V.K., eds.) Reidel (Dordrecht) 529 (1986).

[35] Paczynsky (1986).

[36] Peacock, J. 24th Liege Coll.: Quasars and Gravitational Lenses 86 (1983).

[37] Phinney, E.S. \& Blandford, R.D. Nature 321, 569 (1986).

[38] Press, W.H., \& Gunn, J.E. Astrophys. J. 185397 (1973).

[39] Roberts, D.H. et al Astrophys. J. 293, 356 (1985). 
[40] Schneider, D.P. et al Astrophys. J. 294, 66 (1985).

[41] Schneider, P. Astron. \& Astrophys. 143, 114 (1984).

[42] Shaver, P.A. \& Christiani, S. Nature 321, 585 (1986).

[43] Shaver, P.A., Wampler, E.J., \& Christiani, S. Nature 327, 40 (1987).

[44] Stockton, A. Astrophys. J. 242, L141 (1980).

[45] Surdej, J. et al Astron. \& Astrophys 161, 209 (1986).

[46] Surdej, J. et al Nature 329, 695 (1987).

[47] Turner, E.L., Ostriker, J.P., \& Gott, R.J. Astrophys. J. 2841 (1984).

[48] Turner, E.L., Proc. IAU Symp. 117: Dark Matter in the Universe (Kormendy, J. \& Knapp, G.R., eds.) 227 (1987).

[49] Tyson, J.A., \& Gorenstein, M. Sky \& Telescope 319 (October, 1985).

[50] Vanderreist et al Astron. \& Astrophys 158, L5 (1986).

[51] Walsh, Carswell, \& Weymann Nature 279381 (1979).

[52] Weedman, D.W. et al Astrophys. J. 255, L5 (1982).

[53] Weymann, R.J. et al Nature 285641 (1980).

[54] Young, P., Gunn, J.E., Kristian, J., Oke, J.B. \& Westphal, J.A. Astrophys. J. 241, 507-520 (1980); ibid 244736 (1981b).

[55] Young, P., Gunn, J.E., Kristian, J., Oke, J.B. \& Westphal, J.A. Astrophys. J. 241, 507-520 (1980).

[56] Young, P. et al Astrophys. J. 244, 723; (1981a).

[57] Zwicky, F. Phys. Rev. 51290 (1937). 